\title{
RSAODV : A ROUTE STABILITY BASED AD HOC ON DEMAND DISTANCE VECTOR ROUTING PROTOCOL FOR MOBILE AD HOC NETWORK
}

\author{
Sanjeev K. Prasad ${ }^{1}$ and Karamjit Bhatia $^{2}$ \\ ${ }^{1}$ Ajay Kumar Garg Engg. College, Ghaziabad (India) \\ ${ }^{2}$ Gurukula Kangri Vishwavidyalaya, Haridwar (India)
}

\begin{abstract}
High mobility of mobile nodes is one of the major reasons for link failures in mobile ad hoc network (MANET). The present paper aims at proposing modification of well known Ad hoc On demand Distance Vector $(A O D V)$ routing protocol for MANET seeking stable route for data transmission and incorporating link break avoidance scheme for such networks. The performance of the proposed routing protocol, named as Route Stability based Ad hoc On demand Distance Vector (RSAODV), is evaluated considering some major performance metrics which include average route life time, throughput, packet delivery fraction, normalized routing load and end-to-end delay. The study is based on simulation runs adopting CBR traffic pattern taking care of node failure scenarios. The performance of RAODV is compared with AODV routing protocol. The simulation runs exhibit significant increase in average route life time, packet delivery fraction and throughput for RSAODV, on the other hand, the normalized routing load and end-to-end delay are reduced for this routing protocol as compared to AODV routing protocol.
\end{abstract}

\section{KEYWORDS:}

Route Stability, Node Stability, Node Mobility, Node Energy, Traffic Load, Transmission Power.

\section{INTRODUCTION}

In mobile ad hoc network (MANET), mobile nodes are continuously moving from one location to another with a pause-time. Thus, MANET topology can change often and unpredictably. Excessive node mobility may lead to topology changes before network updates can propagate [1]. Many protocols have been proposed for multihop MANET routing to maintain best effort routes. Route stability mainly relies on route lifetime which, in turn, is related to the route length and the lifetime of each link [2]. In large-scale MANETs, the route stability is very important [6]. The hop count of a route may be large. If a route fails, the procedure of route rediscovery results in an increase in control overhead and end-to-end delay. In high-mobility MANETs, the fast changes in topology increase the complexity of routing [10]. Hence, there is a need to construct a route in which each link has long lifetimes.

This paper addresses two main problems in routing of CBR traffic in MANETs - route stability and route recoverability. To improve route stability in large-scale and high mobility MANETs, the present work proposes a Route Stability algorithm. In the proposed protocol, a route stability estimation method and a path finding algorithm are developed to find and maintain stable routes for communication services in MANETs. In present work, route estimation relies on the node energy, node mobility, transmission power and traffic load. For the route recoverability in high mobility MANETs, the present work proposes a Link Break Avoidance Scheme. The performance of RSAODV is compared with the well-known Ad-hoc On demand Distance Vector (AODV) DOI : $10.5121 /$ ijwmn.2014.6609 
International Journal of Wireless \& Mobile Networks (IJWMN) Vol. 6, No. 6, December 2014

routing protocol [9]. The simulation runs exhibit significant increase in average route life time, packet delivery fraction and throughput for RSAODV where as normalize routing load and endto-end delay are decreased for this routing protocol as compared to AODV routing protocol.

\section{RELATED WORK}

In mobile ad hoc network, nodes are continuously moving from one place to another with a pause-time and stability of link/route is an important parameter in such an environment [5]. Route Stability is the quality which asserts the network environment's consistency. Many stability based routing algorithms considering different criteria have been proposed in literature for MANETs. The power aware and transmission range route stability schemes are proposed in $[3,4,16]$. In [3], AOSV algorithm for computing link/route stability, initially, every node begins to estimate the stabilities of radio links to its neighbours and for keeping track of the link stabilities between a node and its neighbors, each node periodically broadcasts Hello message (HELLO) including the location of the broadcasting node towards its neighbours. PAMAS routing algorithm [4] is based on minimizing the amount of power required to get a packet from source to destination. This algorithm selects routes with more hops than other routing algorithms. In [16], AODVSR is proposed where a node receives Hello messages and it first calculates its distance from the neighboring node using received HELLO messages. Using this distance information, it evaluates the stability of radio link to the broadcasting neighbour. This information is recorded for estimating stabilities of multi-hop routes in follow-up processes. In path discovery process, source node broadcasts RREQ packet that has new link stability field. The intermediate node rebroadcasts only the RREQ packet with the maximum value for route stability among received RREQs. The location updating route stability schemes are proposed in [11, 17]. In [11], the algorithm works on the stability of the node which is determined by the combination of the speed of the node, the range of the node, the location of the node and the battery power of the node. The main focus of the algorithm is to reduce the number of acknowledgment packets by varying the updating information of less stable nodes more frequently as compared to the more stable nodes. An algorithm presented in [17] enables mobile nodes to implement self-regulated movements in MANETs. This scheme maintains a certain level of network-based stability by harmonizing the node movements autonomously due to their limited transmission range and dynamic topology. The work employed entropy methods for forecasting the future moments of node.

The energy aware and traffic load route stability schemes have been presented in [7, 12, 13]. In [7], Tomas Holmberg et. al. estimate the traffic at node level by using the incoming/outgoing packets and introduced traffic estimator that uses two steps in order to predict the traffic over the links in ad hoc networks. In [12], EA-DYMO considers both the traffic load and energy aware path for route stability. The work modifies the route selection procedure of DYMO [22] by taking ratio of the energy factor and the average traffic load for each path. In [13], author has designed a variable range energy aware dynamic source routing in which the route selection is based on energy, stability and traffic load. It selects two routes- main and alternate.

The network depended route stability schemes are discussed in $[14,15]$. Both schemes modify the AODV path discovery phase for finding stable route. In [14], Tamanna et. al. proposed the concept of "moving average" to calculate the neighbor stability. In their work each node calculates the stability of its neighboring nodes considering both old stability and current stability values. The old stability means the stability a node has encountered in the previous stability calculation. Stability calculation is triggered by a timer which expires after a constant interval periodically. In [15], the authors presented a scheme (VON scheme) that depends on the velocity and the traffic load of the nodes to determine the nodes involved in rebroadcast to build a stable 
International Journal of Wireless \& Mobile Networks (IJWMN) Vol. 6, No. 6, December 2014

route. This scheme divides the nodes into high speed nodes and low speed nodes. High speed nodes do not participate in the route discovery phase as they produce unstable routes.

The weight-based stable routing scheme is presented in [2, 18]. In [2], a Stable Weight-Based On-Demand Routing Protocol (SWORP) for MANETs is proposed in which the weight of a route is decided by three factors: the route expiration time, the error count, and the hop count. The route discovery mechanism finds multiple routes from the source node to the destination node. The algorithm selects the path with the largest weight value for routing. In [18], a Link lifetime based Backup Routing (LBR) a modified version of SWORP is reported. LBR also selects the multiple paths with the largest weight value for routing but it keeps the remaining routes as a backup route.

\section{PROPOSED MODEL FOR RSAODV ROUTING PROTOCOL}

The proposed model aims at improving the well known AODV routing protocol. The new protocol is named as RSAODV routing protocol. The aim of RSAODV is to provide a more stable route with avoidance of route break for mobile ad hoc networks. In this scheme, route stability is estimated by the stability of node which depends on node energy, node mobility, transmission power and traffic load. These four major processes are employed to find and maintain the most stable route from source to destination. The mechanism of the proposed RSAODV routing protocol is shown in Fig. 1.

The abbreviation used in model are : RSV - Route Stability Value, NSV - Node Stability Value, UN -Upstream Node, DN - Downstream Node, RT - Routing Table, RM - Route Maintenance, RD - Route Discovery, RREQ packet- Route REQuest packet, RREP packet - Route REPly packet, RM - Route Maintenance, RD - Route Discovery.

\subsection{Route Stability Estimation}

In order to allow more stable routes for data transmission in MANETs, we hereby propose to include a new field, called route stability (RS) field, which is a measure of the stability of the route, in the RREQ packet, the routing table and the HELLO packet of AODV routing protocol. 
International Journal of Wireless \& Mobile Networks (IJWMN) Vol. 6, No. 6, December 2014

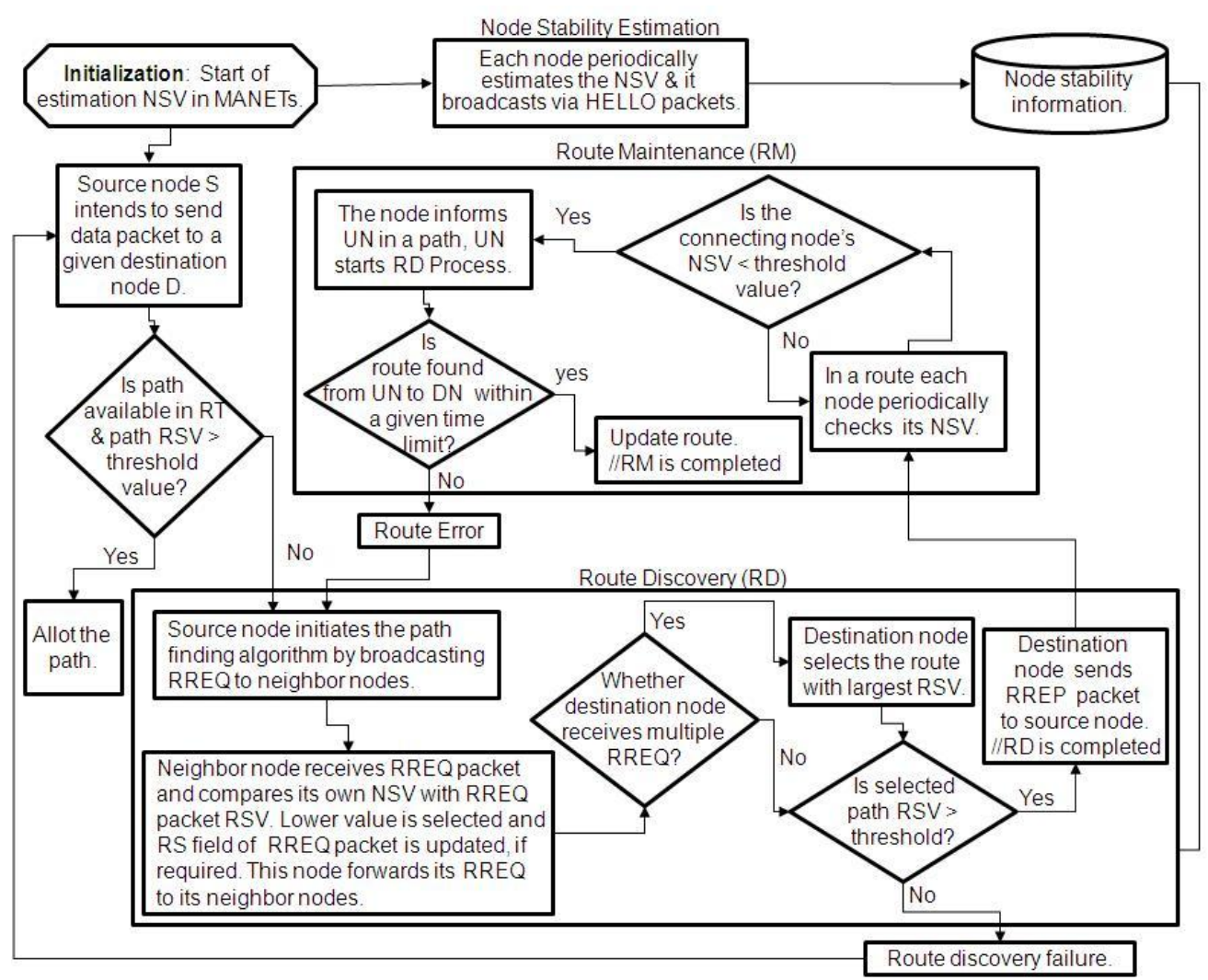

Figure 1. The Mechanism of the Proposed RSAODV Routing Protocol.

In a network, each node periodically broadcasts HELLO message with node stability value. When a node receives HELLO message it checks node stability value of received HELLO packet and if it is larger than the threshold value then the node adds the sender of the HELLO packet as its neighbor node. To determine route stability of the path the source node invokes route discovery process. The proposed model includes following steps for stable route discovery:

1) The source node adds node stability value (NSV) in RS field of RREQ packet and sends it to the neighbor nodes (which may be an intermediate node in the final route).

2) Neighbor node compares its own $N S V$ with received RREQ packet route stability value $(R S V)$. The lowest value is selected i.e. if $N S V<\mathrm{RSV}$ then $R S V \leftarrow \mathrm{NSV}$ in RREQ packet. Forward RREQ packet to the neighbors and so on. This ensures lowest $N S V$ to be the route stability value of the path being discovered.

3) If destination node receives more than one RREQ packet then it sends an RREP packet in response to the RREQ packet with the largest Route Stability value to the source node.

The algorithm 1 shows the determination of minimum Route Stability Value of the path.

Algorithm 1: RS_Value(SN, RN)

$\{/ / S N$ sending node and $R N$ receiving node

If $(N S V[S N]<N S V[R N])$

Update $N S V[S N]$ in RREQ packet. 
International Journal of Wireless \& Mobile Networks (IJWMN) Vol. 6, No. 6, December 2014

Based on the above scheme, the route stability $R S(p)$ of path is equal to the lowest node stability value $N S(n)$ of the node in a path and is exceeding a threshold value $\omega . R S(p)$ is given by following expression:

$$
R S(p)=\operatorname{MIN}(N S(\text { SourceNode }), . ., N S(\text { IntermediateNode }), \ldots, N S(\text { DestinationNode }))
$$

where $R S(p)>\omega$, the selecting value for route stability.

\subsection{Node Stability Estimation}

In our method, the estimation of the node stability $N S(n)$, of a node $n$, is considered to be based on four different processes e.g. Energy Level (EL), Mobility of Node $(M N)$, Transmission Power $(T P)$ and Traffic Load (TL). In our scheme, nodes periodically calculate $N S$ value and transmit it through HELLO message. If any node receives larger NSV than predefined threshold value $\beta$ from some node (say $\mathrm{x}$ ), it adds node $\mathrm{x}$ as its neighbor node. The proposed simple linear equation for node stability $N S_{n}$ of a node $n$ is given by the following expression:

$$
N S(n)=a^{*} E L+b * M N+c^{*} T P+d^{*} T L \quad \text { where } N S(n)>\beta
$$

$\beta$ is the minimum node stability value for the route and $a, b, c$, and $d$ are weighing factors for the corresponding network parameters. All four parameters, with values ranging from 0 to 1 , are chosen so that $a+b+c+d=1$. These values are kept flexible so that they can be changed as per the network scenario. For example, when the network traffic is very high ' $d$ ' can be given more weight than the other three. Similarly, in a high mobility network, the weight ' $b$ ' dominates the other factors.

In the computation of $N S(n)$ above, the $E L$ is energy level of node. When energy of each intermediate node is empty, it is impossible to communicate and link break occur. The $M N$ is the mobility of node that holds the current speed of node. $M N$ implies node stability and hence the stability of route. The $T P$ is the transmission power and used in order to elect the node which can cover the largest range. The $T L$ is traffic load on a node. Nodes, such as intermediate nodes, are engaged with other jobs. Our method also incorporates the provision to avoid such type of nodes at the time of making path.

\subsubsection{Energy Model}

In our energy model, the Energy Level $E L$ of a node $n$ refers to the available energy $E A$ of a node $n$ which depends on total initial energy and consumed energy [12]. Available energy of node $n$ is given by the following expression:

node.

$$
E A(n)=T E(n)-E C(n) \quad \text { where } E A(n)>\varepsilon \text {, the threshold energy level for a }
$$

$T E(n)$ is the total initial energy of node $n$ and $E C(n)$ is the consumed energy of node $n$. The energy consumed $E C(n)$ depends on transmitting $\left(E_{\text {transmit }}\right)$ and receiving $\left(E_{\text {received }}\right)$ one packet is given by following expression :

$$
E C(n)=E_{\text {transmit }}(n)+E_{\text {recieved }}(n)
$$

The energy dissipated in transmitting $\left(E_{\text {transmit }}\right)$ and receiving $\left(E_{\text {received }}\right)$ in one packet and is calculated as follows [13] :

$$
\begin{aligned}
& E_{\text {transmit }}(n)=P_{\text {transmit }}(n) \times T D \\
& E_{\text {recieved }}(n)=P_{\text {received }}(n) \times T D
\end{aligned}
$$


International Journal of Wireless \& Mobile Networks (IJWMN) Vol. 6, No. 6, December 2014

where, $P_{\text {transmit }}$ is a transmitting power of one packet, $P_{\text {received }}$ is a receiving power of one packet and $T D$ denotes the transmission duration of the received/transmit packets at node. In our proposed scheme, the algorithm chooses only those nodes in a route that have higher energy than threshold value $\varepsilon$.

\subsubsection{Node Mobility Factor Deciding Model}

In a dynamic network such as MANET, the mobility of nodes can not be ignored. It has a vital role in maintaining a stable route. Thus, we consider mobility or average displacement of node to be the deciding factor for route setup so that a better route stability can be achieved. In our proposed scheme we adopt the policy given in [19], where a node with lower average displacement has a higher chance of being an intermediate node. The weights assigned to the nodes are reciprocal to their respective displacements. i.e. a node with lower average displacement is assigned a higher weight and the node with higher value is assigned a lower weight. Basically, we consider the mobility of a node by taking the average of the distances covered by mobile node in a given time slot.

We measure the mobility of node $M N_{T}(n)$ as an average speed $S(n)$ of node in time duration $T$. Thus, mobility $M N_{T}(n)$ of node $n$ in time slot $T$ (consisting of $\mathrm{N}$ sub slots) is given by the following expression:

$$
M N_{T}(n)=\frac{\sum_{i=1}^{N} S_{\Delta t_{i}}(n)}{T}
$$

where, $M N_{T}(n)<\alpha$, the threshold mobility value for a node. $S(n)$ is the distance $D(n)$ covered by node $n$ in time interval $\Delta t$. Thus speed of node is given by the following expression :

$$
S_{x}(r)=\frac{Z_{x}(r)}{\Delta x}
$$

where, the distance covered by node $n$ in time slot $\Delta t$ (i. e. from time t-1 to time t) from position $P_{t-1}(\mathrm{x}, \mathrm{y})$ to $P_{t}(\mathrm{x}, \mathrm{y})$ is given by the following expression :

$$
D_{\Delta t}(n)=\sqrt{\left(x(n)_{t}-x(n)_{t-1}\right)^{2}+\left(y(n)_{t}-y(n)_{t-1}\right)^{2}}
$$

\subsubsection{Transmission Power Model}

The transmission power $T P$ is used to elect the node which can cover the largest range. In a dynamic network the transmission power plays a vital role in maintaining the stable route and hence ad-hoc routing protocols must give due emphasis on the radio propagation model for effect on signal strength. The effect not only leads to the average signal power decreasing with the propagation distance but also yields large fluctuation of signal strength in a short range [3], i.e., small-scale fading, or in a medium range, i.e., shadowing. These may result in an unstable link between any two moving nodes even with a short propagation distance. We have used two-ray ground reflection model [21] for the calculation of transmission power of a node. This model considers both the direct path and a ground reflection path for this purpose. In this model the transmission power TP is calculated from the received power $P_{r}$ at distance $d$ and is predicted by : 
International Journal of Wireless \& Mobile Networks (IJWMN) Vol. 6, No. 6, December 2014

$$
P_{r} \mathbb{Q}=\frac{P_{t} G_{t} G_{r} h_{t}^{2} h_{r}^{2}}{d^{4} L}
$$

where, $P_{t}$ is the transmitted signal power, $G_{t}$ and $G_{r}$ are the antenna gains of the transmitter and the receiver respectively and $L(L \geq 1)$ is the system loss. It is common to select $G_{t}=G_{r}=1$ and $L$ $=1$ in $n s$ simulations. $h_{t}$ and $h_{r}$ are the heights of the transmit and receive antennas respectively and $d^{n}$ is the distance between two nodes. We have taken $n=2$ for short distance and $n=4$ long distance in our study.

The two-ray model does not give a good result for a short distance due to the oscillation caused by the constructive and destructive combination of the two rays [20]. Therefore, a cross-over distance $d_{c}$ is calculated in this model. So $d_{c}$ can be calculated as :

$$
d_{c}=\frac{4 \pi h}{\lambda}
$$

where, $\lambda$ is wavelength of signal.

In our scheme if node receiving signal power $P_{r}(d)<\rho$, the threshold value for node signal power, the proposed routing algorithm will not select this node for routing.

\subsubsection{Traffic Load Estimation Model}

Traffic Load for a node is defined as the measure of total cost incurred in sending/receiving packets by a node. If the time-arrival between sending/receiving two packets is $\Delta T$ seconds and the size of packet is $\Delta L$ bits, then the traffic load $T L(n)$ of node $n$ is given by the equation [8] :

$$
T(n)=\frac{\Delta}{\Delta}
$$

In the present work, we have assumed that in the network, each mobile node sends messages to any other node with a uniform rate.

\subsection{Route Discovery}

Route discovery is a process that executes the algorithm 2 to discover the stable route for a requesting source and destination pair. We propose to introduce a new field Route Stability (RS) in $R R E Q$ packet which indicates route stability value of a path from source to particular node. In this process, when network initiates all the nodes are assigned a similar high node stability value (NSV).

\section{Algorithm 2: Route_Discovery}

Step1: The source node $S$ initiates a route discovery process by broadcasting a Route Request (RREQ) packet to all its neighboring nodes. RREQ packet contains the $N S V$ of $S$.

\section{If $(N S V[S]>\beta)$ then}

Else

Broadcast RREQ packet to neighbors with $N S V(\mathrm{~s})$.

Not able to generate RREQ packet.

Step 2: Neighboring node $N$ receives RREQ packet. $N$ may be an intermediate node or a destination node. 
International Journal of Wireless \& Mobile Networks (IJWMN) Vol. 6, No. 6, December 2014

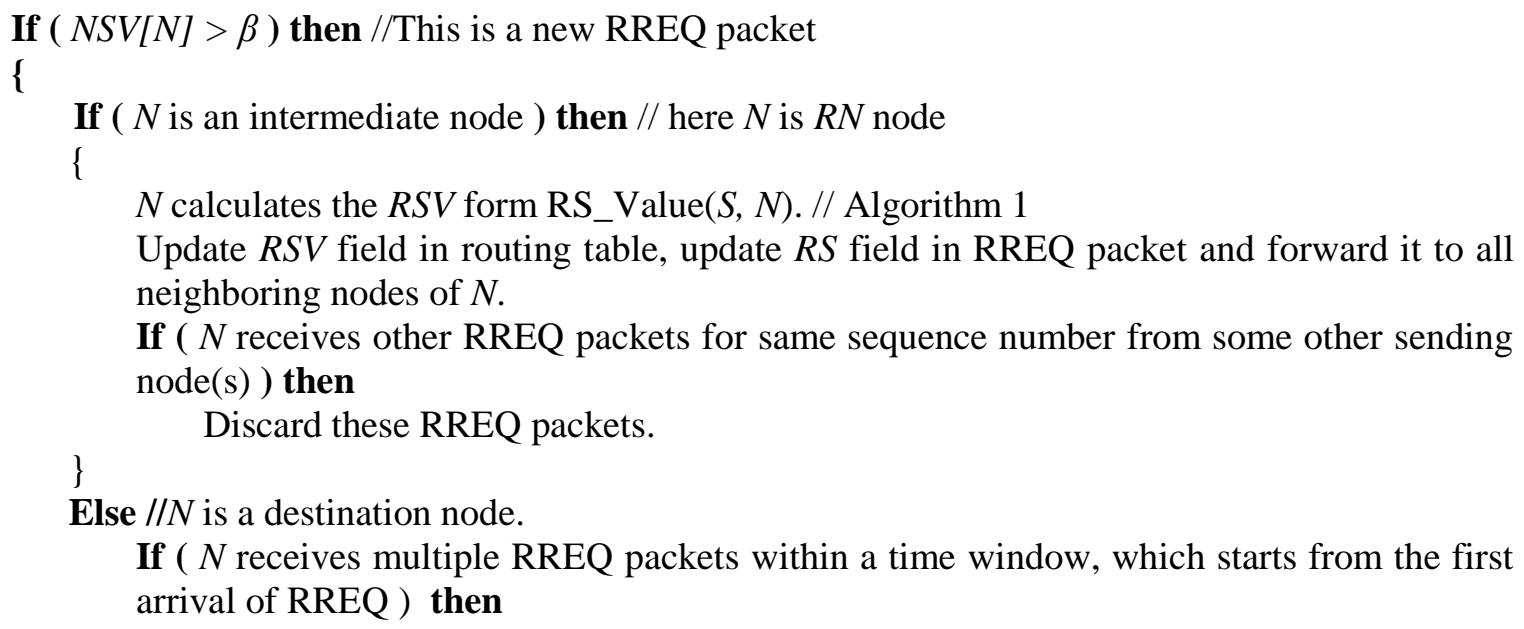

In our model, nodes with $N S V$ greater than threshold value $\beta$ can become the part of active route. Further, the threshold mobility value $\alpha$ of a node has also been considered in the proposed method. This confirms that all the nodes in the active route have sufficient processing power, manageable traffic load and mobility. This implies that all such nodes can't fail too soon resulting in a more stable route.

\subsection{Route Maintenance}

After the stable route is discovered, the source node starts transferring data packets to destination node and periodically checks the stability of the route discovered. At the destination node, the best stability of the discovered route is obtained from received RSVs. During data transmission via stable route, if any intermediate node becomes an unstable node (a node having its NSV less than a predefined threshold value) at some instance of time, or a link/route breakage is detected along this route, the intermediate node informs upstream node which initiates a local route discovery process. If upstream node is unable to find new route, it sends rerouting request (RERREQ) packet back to the source node which then initiates a new route discovery process in order to discover another route to replace the earlier discovered one.

\subsubsection{Link Breaking Avoidance Algorithm}

For the route recoverability, we propose the Link Breaking Avoidance Scheme. In this scheme, each node periodically calculates its $N S V$ and transmits it to its neighbors through hello packets to check the stability of the connecting route. During the transmission of data packets, if any intermediate node's $N S V$ is less than the threshold value $\beta$ then intermediate node $I N$ informs 
International Journal of Wireless \& Mobile Networks (IJWMN) Vol. 6, No. 6, December 2014 upstream node $U N$ through unstable node detection $(U N D)$ packet. $U N$ then reinitiates the route discovery process.

In our route maintenance scheme, we introduce Unstable Node Detection (UND) packet, which is send by the unstable (intermediate) node to its UN, for removal of unstable node. This UND packet contains the sender node address, upstream node $(U N)$ address, downstream node $(D N)$ address, source node address and destination node address.

\section{Algorithm 3: Local_Route_Maintenance}

\section{Step 1:}

If $(I N . N S V<\beta)$ then

$I N$ informs next immediate $U N$ through $U N D$ packet.

\section{Step 2:}

If $(U N . N S V<\beta)$ then

$U N$ declares ROUTE ERROR, $U N$ sends RERR packet to source node \& destination. Source else node reinitiates route discovery process.

//UN checks own neighbor table.

If ( destination node is the neighbor node of $U N$ ) then $U N$ removes $I N$ from the existing path and updates the path.

$U N$ forwards the all data packets to this updated path.

else

$U N$ initiates the route discovery process to the $D N$.

\section{PERFORMANCE EVALUATION}

The performance of proposed RSAODV protocol is evaluated using simulation tool NS-2.35 [20] and is compared with AODV routing protocol. The performance evaluation is done on the basis of following performance parameters :

Packet delivery fraction: The ratio of the number of the successful arrived packets to the number of all packets transmitted by source. The larger value indicates that the more data packets are successfully delivered to destination.

End-to-end delay: The average time spent on data packet transmission from source to destination, including all possible types of delay during the transmission.

Normalized routing load: The normalized routing load is measured by the total number of routing packets sent divided by the number of routing packets delivered successfully.

Throughput: The throughput is defined as the total amount of data a receiver receives from the sender divided by the time it takes for the receiver to get the last packet. The throughput is measured in bits per second (bit/s or bps).

Route lifetime: The route lifetime is defined as the average time elapsed from route establishment to route failure.

\subsection{Performance Evaluation and Result Discussion}

The performance of RSAODV is evaluated through simulation using NS-2.35 [20]. The simulation network area is considered as $1000 \mathrm{~m} \times 1000 \mathrm{~m}$ with 100 nodes in each simulation run 
International Journal of Wireless \& Mobile Networks (IJWMN) Vol. 6, No. 6, December 2014 out of which 49 act as sources and 71 as destinations. For a simulation run, the pause time is set to $50 \mathrm{~s}$ and simulation time is varied from 50s to 500s. Several simulation runs are performed for the performance comparison of both the protocols RSAODV and AODV. The performance comparison of these protocols is done on the basis of packet delivery fraction, end-to-end delay, normalized routing load, throughput and route life time. Table 1 shows the simulation parameters and their values for the study. The comparison results are presented in the pictorial form [Fig. 2a2e].

Table 1: Simulation Parameters

\begin{tabular}{|c|l|l|}
\hline S. No. & Simulation Parameters & Value \\
\hline 1 & Traffic Type & CBR \\
\hline 2 & Number of Nodes & 100 \\
\hline 3 & Pause Time & $50 \mathrm{~s}$ \\
\hline 4 & Simulation Time & $50 \mathrm{~s}$ to500 s \\
\hline 5 & Total Sources and Connections & 49 and 71 respectively \\
\hline 6 & Maximum Speed of Nodes & $20 \mathrm{~m} / \mathrm{s}$ \\
\hline 7 & Area of Network & $1000 \mathrm{~m} \times 1000 \mathrm{~m}$ \\
\hline 8 & Packet Rate & $10 \mathrm{pkts} / \mathrm{s}$ \\
\hline 9 & Packet Size & 512 byte \\
\hline 10 & Mobility Model & Random Mobility Model \\
\hline
\end{tabular}

Packet Delivery Fraction: Fig. 2a shows the comparison of RSAODV and AODV on the basis of packet delivery fraction. It is observed that RSAODV and AODV have almost same packet delivery fraction for simulation run time equal to 50s and as the simulation time increases there is a drop in packet delivery fraction for both the protocols but the packet delivery fraction is significantly higher for RSAODV as compared to AODV. During all simulation runs minimum packet delivery fraction achieved for RSAODV is $86.65 \%$ whereas for AODV it is $81.34 \%$. In AODV routing protocol, when active route fails, the interface queue becomes full. This results into a higher packet drop rate. To overcome this situation, proposed protocol RSAODV selects the node in a path which has less traffic and sufficient energy. This mechanism reduces the chances of route failure thereby improving packet delivery efficiency.

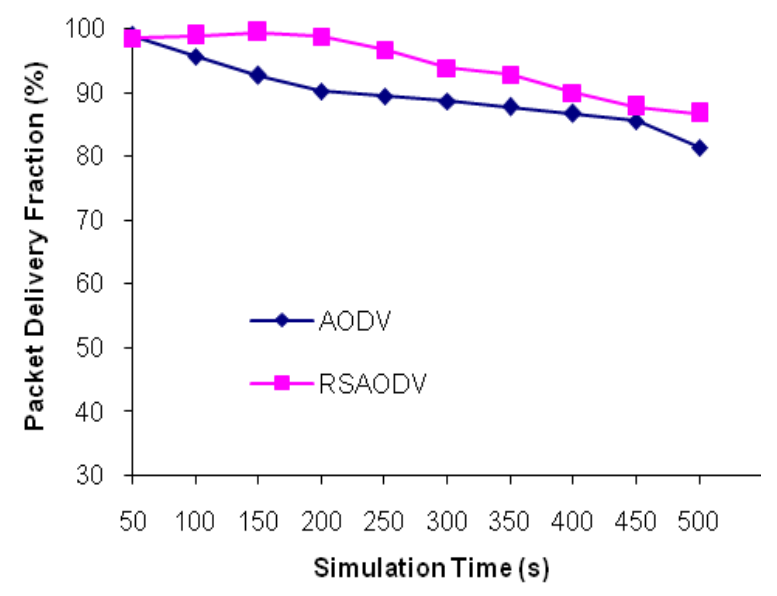

Figure 2a. Packet Delivery Fraction.

End-to-End delay: Fig. $2 \mathrm{~b}$ shows the performance comparison of RSAODV and AODV protocols for end-to-end delay parameter. The prime reasons for end-to-end delay are link failure 
International Journal of Wireless \& Mobile Networks (IJWMN) Vol. 6, No. 6, December 2014

and greater traffic load on intermediate nodes. In AODV, when an active link fails the local route repair or re-route discovery process is initiated. During this period all data packets wait in a queue thereby enhancing delay. The proposed protocol RSAODV incorporates the concept of 'make before break' to avoid such a situation. Here, if a node in active path has NSV less than the threshold NSV, then it informs the upstream node to discover the new route. This mechanism reduces waiting time of data packets resulting lesser end-to-end delay. Traffic load for a node also has a significant effect on the end-to-end delay. In RSAODV, a node having less traffic is selected to be an intermediate node during the path selection process whereas there is no such type of mechanism in AODV for a node selection. As a result of this mechanism in RSAODV, proposed protocol exhibits better performance as compared to AODV for end-to-end delay.

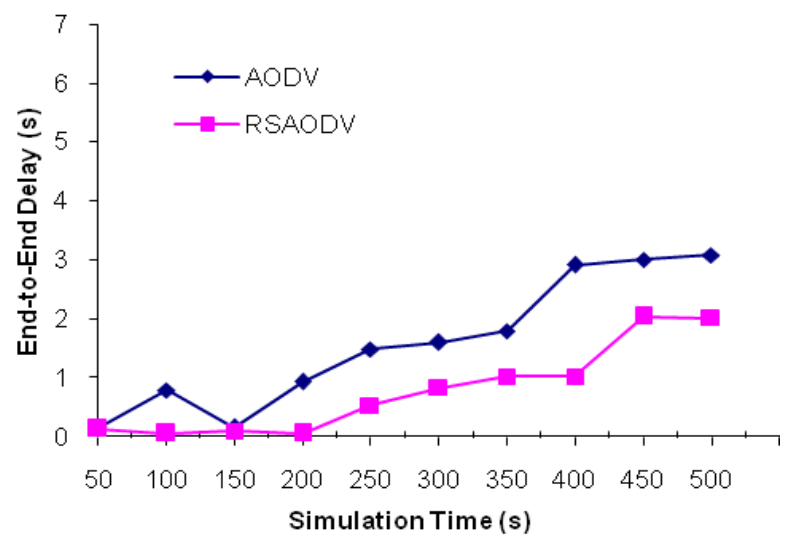

Figure 2b. End-to-End Delay.

Normalized Routing Load: Fig. 2c presents normalized routing load for RSAODV and AODV protocols. The simulation results shows that RSAODV has a lower normalized routing load as compared to AODV. Better performance of RSAODV is due to the lesser number of route breaks in RSAODV as compared to those in AODV resulting in to lesser number of control packets flow in the network.

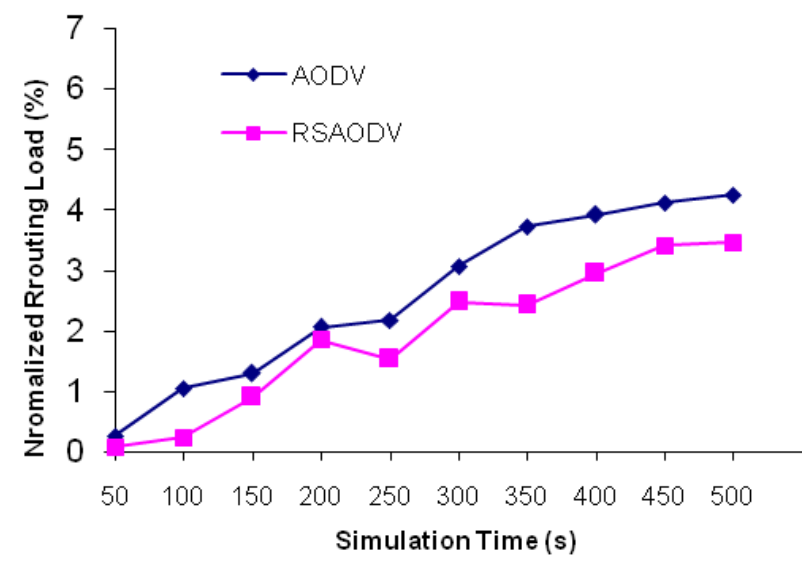

Figure 2c. Normalized Routing Load.

Throughput: Fig. 2d shows comparison of RSAODV and AODV protocols on the basis of throughput measured in kilobits per second (kbps). The data rate of CBR traffic pattern is 10 
International Journal of Wireless \& Mobile Networks (IJWMN) Vol. 6, No. 6, December 2014

packets/sec and the size of each packet is 512 bytes, thus a node transmits at $5 \mathrm{Kbps}$ data rate. The RSAODV achieves higher throughput as compared to AODV in all simulation trials. This is, again, due to incorporated mechanisms in RSAODV as fewer path breaks occur during data packet transmissions in RSAODV as compared to those in AODV.

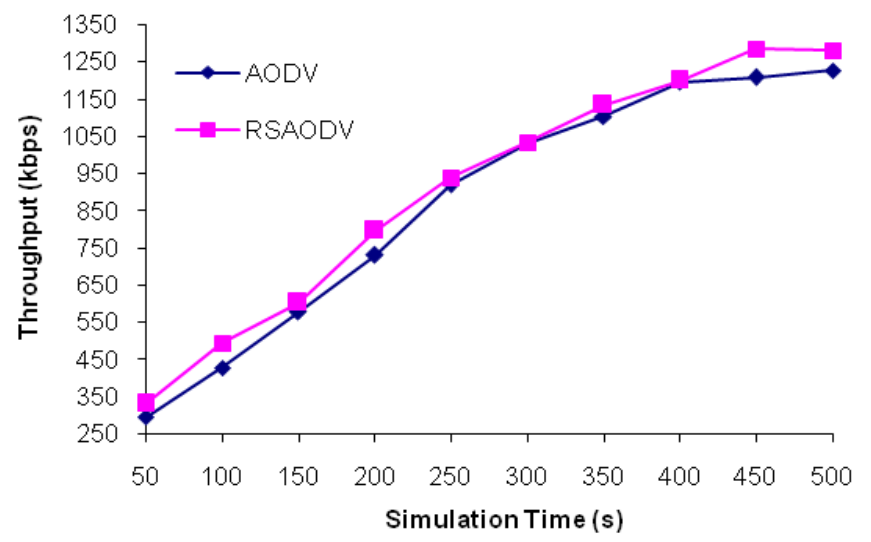

Figure 2d. Throughput.

Route Life Time: Fig. 2e depicts route life time for RSAODV and AODV protocols. The proposed protocol RSAODV exhibit higher route life time as compared to AODV reflecting establishment of more stable routes. The route life time is affected by the energy and speed of all the nodes in a path. The route may fail if the energy of any of the intermediate node in a route goes down due to more control packet transmissions or retransmissions of data packets. The other reason of route failure is the movement of an intermediate node out from the transmission range of other nodes involved in the route. RSAODV algorithm selects those nodes in the path which has good energy and low mobility in the network. RSAODV takes into consideration the traffic in the network in route generation resulting into less numbers of route failures. This enhances route life time for data communication as depicted by Fig. 2e.

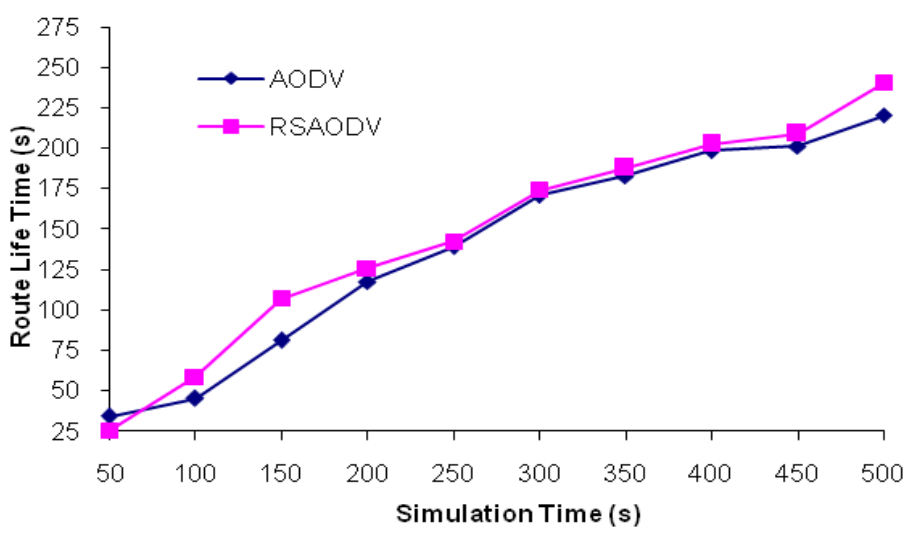

Figure 2e. Route Life Time.

\section{CONCLUSION}

This paper deals with the problem of unstable route for MANETs and presents a new route-stable routing protocol RSAODV. Adding the route stability field to the RREQ packet in routing protocol avoids selection of unstable routes automatically during establishing a route. A make before break concept is proposed in the route repair mechanism of RSAODV. This is 
International Journal of Wireless \& Mobile Networks (IJWMN) Vol. 6, No. 6, December 2014

incorporated by introducing UND packet in RSAODV routing protocol. In the proposed routing protocol a new route is found, as far as possible, before a route break occurs instead of initiating a new source routing discovery as is done in AODV routing protocol. These provisions lead to an improvement in AODV routing protocol. The study is based on several simulation runs considering different performance evaluation metrics with varying pause times. We analyzed the performance of AODV and RSAODV routing protocols on the basis of these metrics which include packet delivery fraction, end-to-end delay, normalized routing load, throughput and route life time. The simulation runs exhibit better results in favor of RSAODV protocol as compared to AODV protocol. This is due to the proposed provisions which not only reduce the packet loss rate and the end-to-end delay but also enhance the utilization of the network resources increasing the throughput and average route lifetime.

\section{REFERENCES}

[1] Larry C. Llewellyn, Kenneth M. Hopkinson and Scott R. Graham, (2011). Distributed Fault-Tolerant Quality of Wireless Networks. IEEE Transactions On Mobile Computing, 10(2), pp175 - 190.

[2] Nen Chung Wang, Jhu Chan Chen. (2006). A Stable On-Demand Routing Protocol for Mobile Ad Hoc Networks with Weight-Based Strategy. Seventh International Conference on Parallel and Distributed Computing, Applications and Technologies, pp166-169.

[3] Jenn-Hwan Tamg, Bing-Wen Chuang, and Fang-Jing Wu. (2006). A Radio-Link Stability-based Routing Protocol for Mobile Ad Hoc Networks. IEEE International Conference on Systems, Man, and Cybernetics 5, pp3697-3701.

[4] Suresh Singh, Mike Woo and C. S. Raghavendra. (1998). Power-Aware Routing in Mobile Ad Hoc Networks. Forth ACM/IEEE International Conference on Mobile Computing and Networking, pp181-190.

[5] Geetha Nair and Dr. N.J.R. Muniraj. (2012). Prediction based Link Stability Scheme for Mobile Ad Hoc Networks. International Journal of Computer Science, 9(6), pp401-408.

[6] Wenjing Yang, Xinyu Yang, Shusen Yang, Yage Gong. (2011). Improving Route Stability in Mobile Ad Hoc Networks Based on Link Lifetime. Journal of Communications, 6( 3), pp205-214.

[7] Tomas Holmberg, Jimmi Grönkvist, Jan Nilsson, and Mattias Sköld. (2007). Traffic Estimation in Mobile TDMA-based Ad Hoc Networks. The Sixth Annual Mediterranean Ad Hoc Networking WorkSh. Corfu, Greece, pp85-91.

[8] Maysam Hedayati, Hamid Reza Hoseiny, Seyed Hossein Kamali and Reza Shakerian. (2010). Traffic Load Estimation And Load Balancing In Multipath Routing Mobile Ad-Hoc Networks. IEEE International Conference On Mechanical and Electrical Technology, pp117-221.

[9] Perkins CE, Royer EM. Ad hoc on-demand distance vector routing. (1999). IEEE Workshop on Mobile Computing Systems and Applications, pp90-100.

[10] Hossam Hassanein, Hongyan Du and Chihsiang Yeh. (2004). Robust Route Establishment in HighMobility MANETs. International Computer Engineering Conference, pp74-80.

[11] Sudip Misra, Sanjay K. Dhurandher, Mohammad S. Obaidat, Namit Nangia, Nitin Bhardwaj, Pankaj Goyal, and Sumit Aggarwal. (2008). Node Stability-Based Location Updating in Mobile Ad-Hoc Networks. IEEE Systems Journal, 2(2), pp237-247.

[12] Sumit Kumar Singh, Shiva Prakash and Kapil Kumar. (2011). Energy Aware Dynamic MANET Ondemand (EA-DYMO). International Journal of Computer Applications, 25(11), pp12-16.

[13] Shiva Prakash, S.C. Gupta, J. P. Saini, Sandip and Vijay. (2013). Design and Implementation Of Variable Range Energy Aware Dynamic Source Routing Protocol for Mobile Ad Hoc Networks. International Journal of Computer Engineering and Technology. 4(1), pp105-123.

[14] Tamanna Afroze, Saikat Sarkar, Aminul Islam and Asikur Rahman. (2009). More Stable Ad-hoc OnDemand Distance Vector Routing Protocol. 4th IEEE Conference on Industrial Electronics and Applications, pp150-155.

[15] Yaser Khamayseh, Ghadeer Obeidat and Asmahan Abu Alhassan. (2009). Enhanced VON-AODV Based on Delayed Rebroadcast Scheme. 4th ACM workshop on Performance monitoring and measurement of heterogeneous wireless and wired networks, pp78-83. 
International Journal of Wireless \& Mobile Networks (IJWMN) Vol. 6, No. 6, December 2014

[16] T. Arul Doss Albert Victorie and Saleem. (2010). Stability based Route Repairing Algorithm for Ad Hoc On- Demand Distance Vector Routing. IEEE International Conference on Industrial and Information Systems, pp98-101.

[17] Sang-Chul Kim. (2011). Self-Regulated Decision for Stable MANET Node Movement. IEEE International Conference on Ubiquitous and Future Networks. pp185 - 190.

[18] Wenjing, Xinyu and Shusen. (2009). A Stable Backup Routing Protocol Based on Link Lifetime in Mobile Ad hoc Networks. 3rd IEEE International Conference on Mobile Ubiquitous Computing, Systems, Services and Technologies, pp202-207.

[19] Suchismita Chinara, and Santanu Kumar Rath. (2008). Energy Efficient Mobility Adaptive Distributed Clustering Algorithm for Mobile Ad Hoc Network. 16th IEEE International Conference on Advance Computing and Communication, pp265-272.

[20] NS-2, The ns Manual, http: //www. isi.edu/nsnam/ns.

[21] T. S. Rappaport. (1996). Wireless Communications, Principles and Practice. Prentice Hall.

[22] I. D. Chakeres and C. E. Perkins. (2007). Dynamic MANET On demand (DYMO) routing. Internetdraft. Draft-ietf-manetdymo-11. http://www.ietf.org/internet-drafts/draft-ietfmanet-dymo-11.txt. Mobile Ad hoc Networks Working Group, November 2007.

\section{Authors}

Sanjeev Kumar Prasad is an Assistant Professor in Department of Computer Application at Ajay Kumar Garg Engineering College, Ghaziabad, an affiliated college of Uttar Pradesh Technical University, Lucknow, (India). He received M. Tech. degree in Computer Science \& Engineering from Uttar Pradesh Technical University, Lucknow, (India) and he is pursuing Ph.D. in Computer Science from Gurukula Kangri Vishwavidyalaya, Haridwar, (India). Before

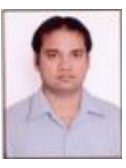
joining this college, he has served many other colleges affiliated to Uttar Pradesh Technical University, Lucknow, (India). He has got published 4 papers in international journals and 3 papers published in international proceeding conferences. His areas of interest include Mobile Ad-Hoc Networks and Computer Graphics.

Dr. Karamjit Bhatia is a Professor in Department of Computer Science at Gurukula Kangri Vishwavidyalaya, Haridwar (India). He has got published 27 papers in conference proceedings/ Journals of repute etc. He got his Masters degree in Physics from Garhwal University, Srinagar, Garhwal (India) in 1986 and obtained his M. Phil. degree in Computer Applications from University of Roorkee (now IIT), Roorkee (India) in 1988. He was

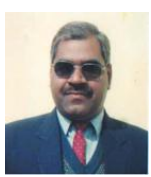
awarded Ph.D. in Computer Science from Gurukula Kangri Vishwavidyalaya, Haridwar (India) in 2001. He has taught several papers at Under Graduate and Post Graduate levels. His areas of interest include Distributed Systems, Mobile Ad-hoc Networks and Digital Image Processing. 